chlorhexidine-based products, ensuring microbiological quality of the finished product in addition to patient safety, so that similar outbreaks can be prevented.

Acknowledgments. The authors thank Dr Janet W. Reid for the English text review.

Financial support. This study was financed in part by the Coordenação de Aperfeiçoamento de Pessoal de Nível Superior, Brazil (CAPES), Finance Code 001. These government funds covered only the cost of laboratory materials and had no role in the study design or the decision to submit the work for publication.

Conflicts of interest. All authors report no conflicts of interest relevant to this article.

\section{References}

1. Maes M, Higginson E, Pereira-Dias J, et al. Ventilator-associated pneumonia in critically ill patients with COVID-19. Crit Care Lond Engl 2021;25:25.

2. Blonz G, Kouatchet A, Chudeau N, et al. Epidemiology and microbiology of ventilator-associated pneumonia in COVID-19 patients: a multicenter retrospective study in 188 patients in an un-inundated French region. Crit Care 2021;25:1-12.
3. Hocková B, Riad A, Valky J, et al. Oral complications of ICU patients with COVID-19: case-series and review of two hundred ten cases. J Clini Med 2021;10:581.

4. Tavares M, Kozak M, Balola A, Sá-Correia I. Burkholderia cepacia complex bacteria: a feared contamination risk in water-based pharmaceutical products. Clin Microbiol Rev 2020;33:1-25.

5. Shaban R, Sotomayor-Castillo C, Nahidi S, et al. Global burden, point sources, and outbreak management of healthcare-associated Burkholderia cepacia infections: an integrative review. Infect Control Hosp Epidemiol 2020;41:777-783.

6. Saalfeld SMS, Shinohara DR, Szczerepa MMA, et al. Consecutive outbreaks of Burkholderia cepacia complex caused by intrinsically contaminated chlorhexidine mouthwashes. Am J Infect Control 2020;48:1348-1353.

7. Silbert S, Pfaller MA, Hollis RJ, et al. Evaluation of three molecular typing techniques for nonfermentative gram-negative bacilli. Infect Control Hosp Epidemiol 2004;25:847-851.

8. Leong LEX, Lagana D, Carter GP, et al. Burkholderia lata infections from intrinsically contaminated chlorhexidine mouthwash, Australia, 2016. Emerg Infect Dis 2018;24:2109-2111.

9. Osman H, Nguyen P. First case of COVID-19 complicated with Burkolderia cepacia pneumonia and bacteremia. Chest 2020;158:A544.

10. Yang S, Hua M, Liu X, et al. Bacterial and fungal coinfections among COVID-19 patients in intensive care unit. Microbes Infect 2021;104806.

\title{
A practical approach to defining aerosol-generating procedures
}

\author{
Moi Lin Ling MBBS, FRCPA, CPHQ, MBA ${ }^{1}$ (1), Alex R. Cook PhD², Kalisvar Marimuthu MRCP ${ }^{3}$, Surinder Pada FRACP4, \\ Brenda Ang $\mathrm{MPH}^{5}$, Koh Cheng Thoon $\mathrm{MRCPCH}^{6}$, Meow Ling Foo $\mathrm{MPH}^{7}$, Xin Yi Ong $\mathrm{MPH}^{8}$, Adelina Young $\mathrm{MPH}^{8}$ and \\ Dale Fisher FRACP ${ }^{9,10}$ \\ ${ }^{1}$ Infection Prevention and Epidemiology, Singapore General Hospital, Singapore, ${ }^{2}$ Saw Swee Hock School of Public, National University of Singapore and \\ National University Health System, Singapore, ${ }^{3}$ Infectious Diseases, National Centre of Infectious Diseases, Singapore, ${ }^{4}$ Infectious Diseases, Ng Teng Fong \\ General Hospital, Singapore, ${ }^{5}$ Infectious Diseases, Tan Tock Seng Hospital, Singapore, 'Infectious Diseases, KK Women's and Children's Hospital, Singapore, \\ ${ }^{7}$ Infection Control, Khoo Teck Puat Hospital, Singapore, ${ }^{8}$ Patient Safety and Quality Improvement Branch, Singapore Ministry of Health, Singapore, ${ }^{9}$ Division of \\ Infectious Diseases, National University Hospital of Singapore, Singapore and ${ }^{10}$ Department of Medicine, Yong Loo Lin School of Medicine, National University of \\ Singapore, Singapore
}

To the Editor-The use of appropriate personal protective equipment $(\mathrm{PPE})$ is crucial in preventing transmission of SARS-CoV-2 to healthcare workers (HCWs) when caring for COVID-19 patients. However, the debate on the importance of different modes of transmission of SARS-CoV-2 continues, and it affects the type of PPE recommended for use. ${ }^{1}$ Moreover, with this pandemic still in progress, the issue of conserving PPE is a practical dilemma. ${ }^{2}$ Clinicians are naturally concerned if they are asked to undertake a procedure potentially generating aerosols while using droplet precautions. Countries and specialist societies define and specify the list of aerosol-generating procedures (AGPs) differently. In practical terms, a risk assessment is needed for some procedures with borderline risk such as nasogastric tube insertion. Although the recommendations of PPE for known or suspect COVID cases is clearer, there is greater uncertainty regarding precautions for nonsuspect cases, especially in high-prevalence settings.

Author for correspondence: Moi Lin Ling, E-mail: ling.moi.lin@singhealth.com.sg. Or Prof Dale Fisher, E-mail: mdcfda@nus.edu.sg

Cite this article: Ling ML, et al. (2022). A practical approach to defining aerosolgenerating procedures. Infection Control \& Hospital Epidemiology, 43: 1083-1084, https://doi.org/10.1017/ice.2021.184
The Singapore National Infection Prevention and Control Committee developed a list of "Procedures of Concern" (Table 1) to help HCWs identify procedures of higher risk that require additional measures to prevent transmission from unidentified COVID-19 cases in the hospital for other reasons. The risk is dependent on the procedure, community prevalence of COVID-19, proportion of diagnosed and isolated infections, and healthcare facility-level screening.

Procedures of concern are defined as any medical procedure that can induce the production of aerosols of various sizes, including small $(<5 \mu \mathrm{m})$ particles containing SARS-CoV-2. For all other procedures, standard precautions should apply. During periods of low community prevalence, the emphasis should be on the use of standard precautions. ${ }^{3}$ In general, AGPs and procedures of concern should be avoided in patients with suspected or confirmed cases of COVID-19, unless urgently required. Ideally, these should be performed in an airborne infection isolation room (AIIR) whenever possible. ${ }^{4}$ When unavailable and the procedure must occur in situ (eg, intubation during resuscitation), staff are advised to draw the privacy curtains and remove any shared equipment, supplies, or linen from the immediate vicinity prior to performing the AGP. In addition, the number of HCWs who are 
Table 1. Procedures of Concern

\begin{tabular}{ll}
\hline - Tracheotomy/tracheostomy & - Bronchoscopy \\
procedures including suctioning, & - Noninvasive ventilation \\
cleaning and changing & - Pulmonary function tests \\
tracheostomy devices & - Nebulised medications via simple \\
- Manual ventilation, & face mask \\
eg, bag-valve-mask & - High-flow nasal oxygen \\
- Intubation/Extubation & - Sputum induction using \\
- Open suctioning where a & hypertonic saline \\
single-use catheter is inserted & - Insertion and removal of \\
into ETT or tracheostomy & laryngeal mask airway \\
- Dental procedures requiring use & - Endoscopy involving upper GI \\
of high speed drills & tract, eg, OGD, ERCP \\
- Upper ENT airway procedures, & - Surgeries involving high-speed \\
eg, nasoendoscopy, & drills/cutting in the respiratory \\
transphenoidal surgery & tract or paranasal sinuses \\
- Thoracic surgeries, airway/lung & - Facial fracture fixation with drills, \\
parenchymal breach, & intra oral procedures with \\
pneumonectomy/NATS/ & suctioning \\
endobronchial surgery (CABG & - Plastics and maxillofacial \\
and cardiac surgery excluded) & surgeries involving upper \\
& respiratory system \\
\hline
\end{tabular}

Note. ETT, endotracheal tube; ENT, ear-nose-throat; VATS, video-assisted thoracoscopic surgery; CABG, coronary artery bypass grafting; GI, gadstrointestingal; OGD, oesophagogastro-duodenoscopy; ERCP, endoscopic retrograde cholangiopancreatography.

present in the patient's room during an AGP should be limited to only those necessary to safely perform or assist in the procedure.

At some stage in the pandemic, there is a point of community prevalence at which the risk of an asymptomatic COVID-19 case presenting for an AGP increases sufficiently that all patients should be regarded as suspects for the purposes of infection prevention and control and contrariwise as the epidemic wanes. Because the acceptable risk may differ by procedure, our recommendation is to couple a country-specific per-patient risk of undiagnosed SARS-CoV-2 infection, $\pi$, derived from epidemiological data, with a procedure-specific risk threshold, $\theta_{p}$. When $\pi<\theta_{p}$, nonpandemic guidelines can apply for procedure $p$, but when $\pi \geq \theta_{p}$, pandemiclevel measures should be implemented. Although the risk $\pi$ of a patient having undiagnosed infection is not known, a conservative upper bound can be derived. Seroprevalence in mid-2020 suggested 10 -fold more infections than cases, though this has probably fallen as more testing has been done. ${ }^{3}$ Assuming an infectious period of 10 days and, conservatively, that all infections are asymptomatic, the number of asymptomatic infections in the community would be 100 times the daily incidence of newly diagnosed cases. For instance, if the daily incidence of diagnosed COVID-19 is 200 per day in a country of 20 million and there have been 2,000 diagnosed cases in the previous 10 days and at most 20,000 undiagnosed but infectious individuals, up to 1 in 1,000 patients would be expected to be asymptomatically infected $(\pi=0.1 \%)$. For an AGP with greater risk, the threshold might be set at 1 in 100,000, in which case the incidence would be sufficiently high to warrant pandemic measures. For lower-risk procedures, standard measures could be considered. The rule of thumb (multiplying diagnosed cases by 100) is deliberately simple and in most settings conservative because, in reality, symptomatic cases are likely to be identified through screening and case detection is likely to have improved over the first year of the pandemic.

We report a practical approach to assist HCWs in determining risks and identifying procedures of concern. There are still gaps in evidence regarding SARS-CoV-2 transmission routes, risk to HCWs, and safety of AGPs. ${ }^{5}$ PPE guidelines written in the context of an evidence gap and of high professional anxiety may cause unnecessary waste of PPE. Moreover, necessary clinical interventions may be unnecessarily cancelled which may result in poorer outcomes for patients. Areas experiencing high rates of transmission should be necessarily conservative in protecting HCWs undertaking procedures of concern. At this stage of the pandemic and moving forward, many countries have or will have improving prevalence, and some guidance to de-escalate infection prevention and control measures for procedures in nonsuspected cases will be needed.

\section{Acknowledgments.}

Financial support. No financial support was provided relevant to this article.

Conflicts of interest. All authors report no conflicts of interest relevant to this article.

\section{References}

1. Wilson NM, Norton A, Young FP and Collins DW. Airborne transmission of severe acute respiratory syndrome coronavirus-2 to healthcare workers: a narrative review. Anaesthesia 2020. doi: 10.1111/anae.15093.

2. COVID-19 coronavirus pandemic. Worldometer website. https://www. worldometers.info/coronavirus/\#countries. Accessed March 19, 2021.

3. Infection prevention and control during health care when coronavirus disease (COVID-19) is suspected or confirmed. Interim guidance 29 June 2020. World Health Organization website. https://apps.who.int/iris/bitstream/ handle/10665/332879/WHO-2019-nCoV-IPC-2020.4-eng.pdf. Accessed March 19, 20210.

4. Pollán M, Pérez-Gómez B, Pastor-Barriuso R, et al. Prevalence of SARSCoV-2 in Spain (ENE-COVID): a nationwide, population-based seroepidemiological study. Lancet 2020 396:535-544.

5. Jayaweera M, Perera H, Gunawardana B, Manatunge J. Transmission of COVID-19 virus by droplets and aerosols: a critical review on the unresolved dichotomy. Environ Res 2020;88. doi: 10.1016/j.envres.2020.109819. 